\section{An Exploration of} the Research

Trends in the

Digital Library

Evaluation

\section{Domain}

Giannis Tsakonas, Angelos

Mitrelis, Leonidas

Papachristopoulos, Christos

Papatheodorou

Dept. of Archives and Library

Science, Ionian University,

Greece

\section{Summary}

In this poster we present the research trends that governed the Digital Library evaluation field within the decade 20012010 in the JCDL and ECDL

conferences. The DL evaluation literature was annotated using the domain ontology DiLEO, which defines explicitly the main concepts of the digital library evaluation field and their correlations.

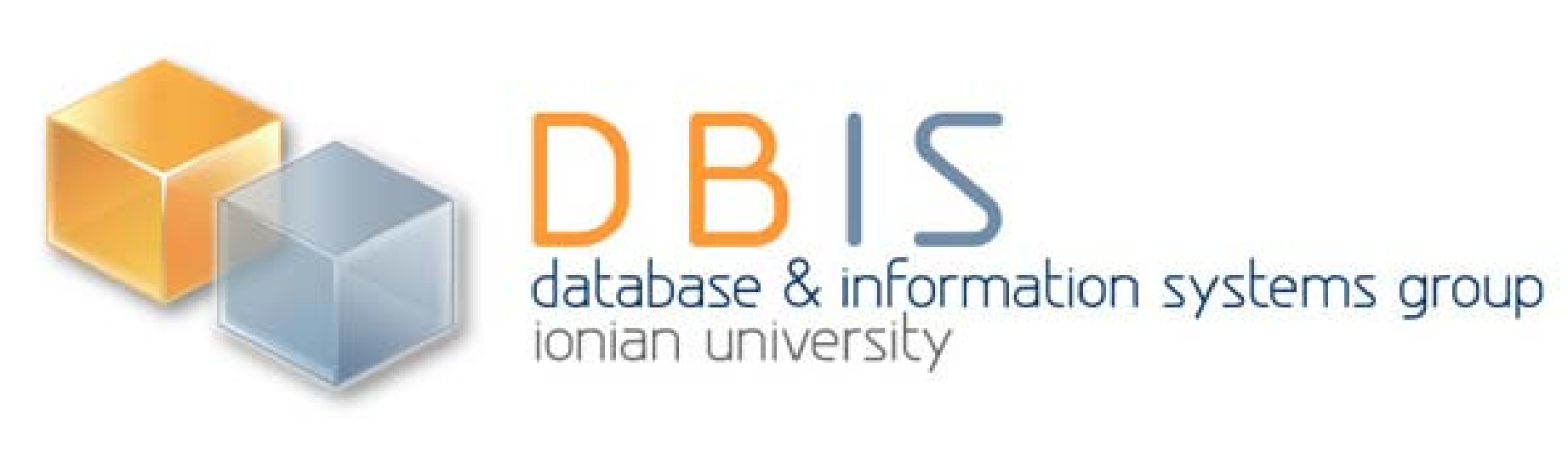

Poster in 12th ACM/IEEE-CS Joint Conference on Digital Libraries, June 1014, 2012, Washington, DC, USA.

\section{Instrument for Semantic Annotation}

DiLEO, the domain ontology for digital library evaluation.

Investigated:

- 5 classes

- 5 properties

- 3698 triples (domain class property - range class)

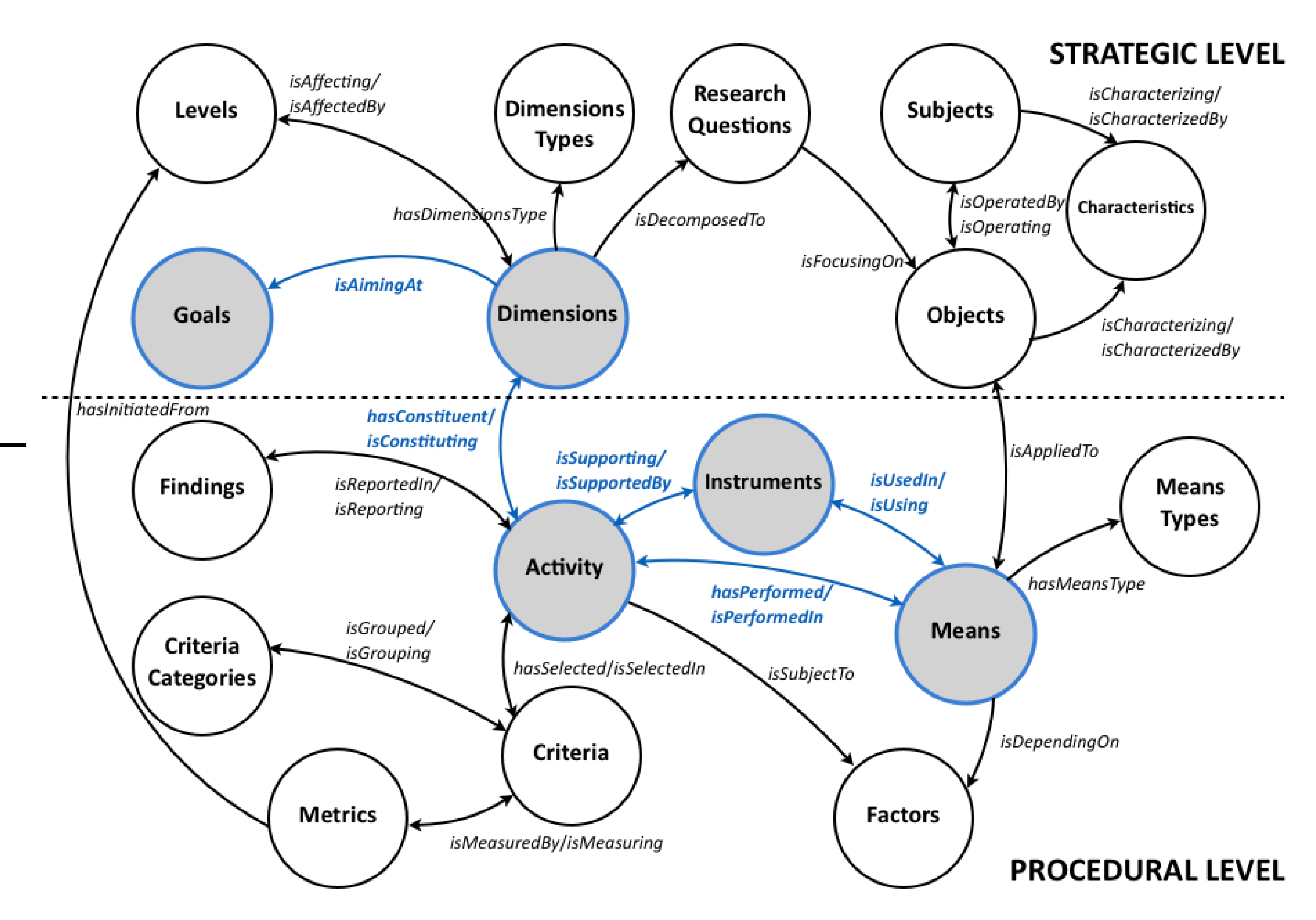

\section{Process and Statistics}

To identify the papers with DL evaluation interest, two domain experts worked independently. Their decisions were based on a triple of common elements: (a) title, (b) abstract and (c) author keywords.

Disputes between the experts were resolved with the intervention of a third researcher who provided additional ratings.

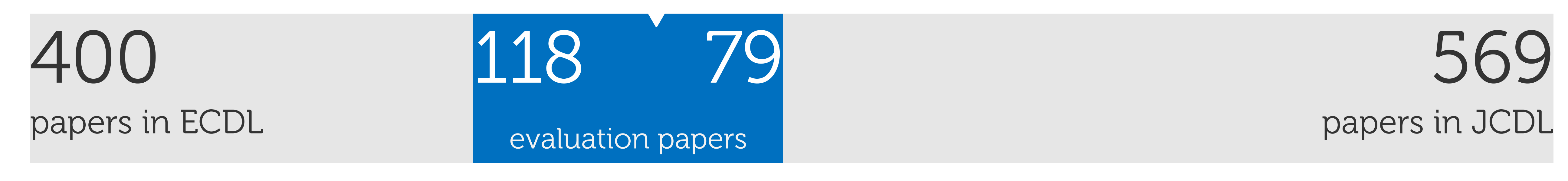

\section{Analysis}

A weighted undirected graph was created to highlight the significance of concepts and their in-between associations. The nodes correspond to DiLEO subclasses. The edges refer to the properties associating them. The betweenness centrality (CB) of the nodes reflect the DiLEO subclasses' significance.

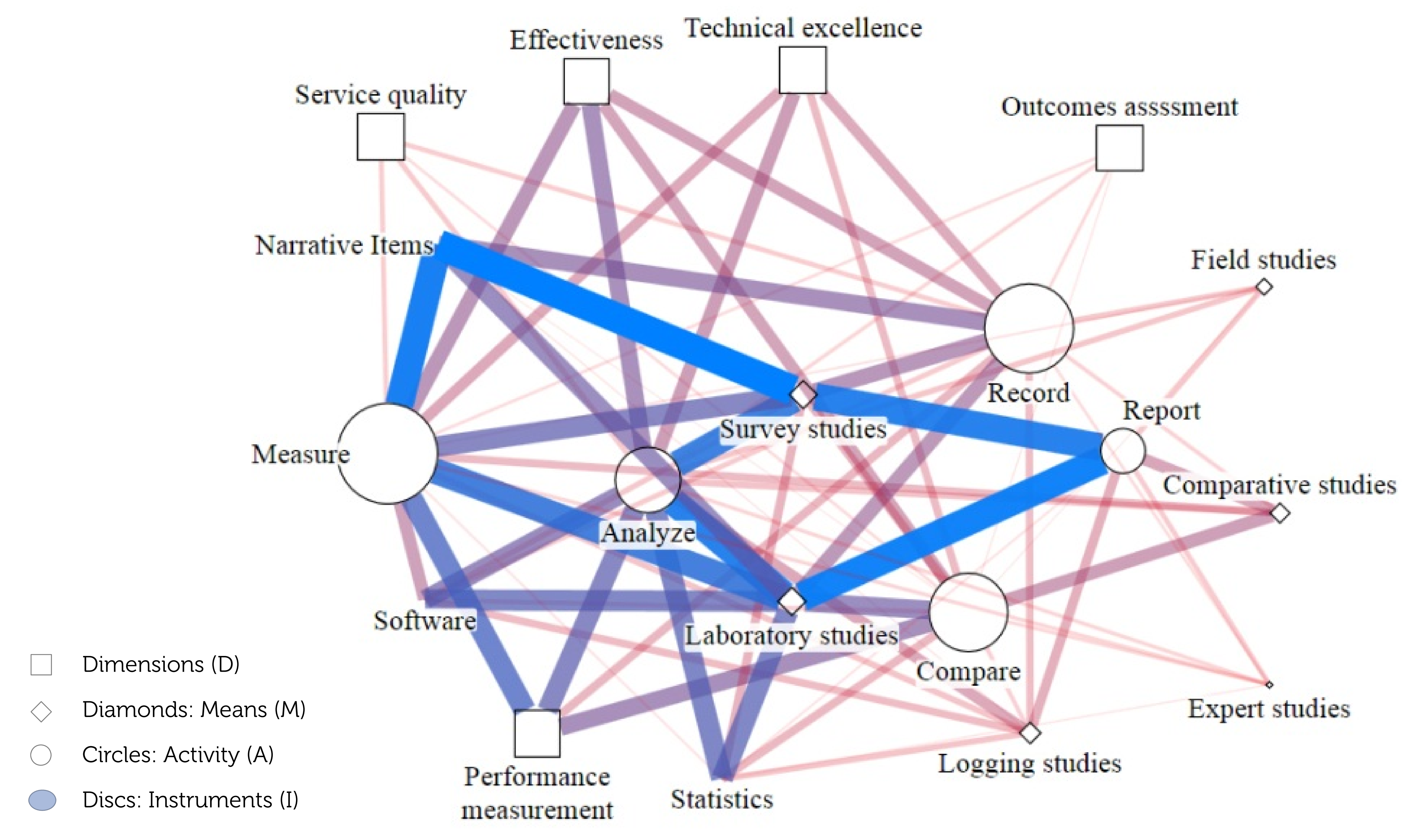

\section{Findings}

All Dimensions subclasses share the same centrality $(C B=13.71)$ for both conferences.

Most significant Means are Laboratory and Survey Studies $(C B=8.69)$, followed by Comparison $(\mathrm{CB}=6.44)$ and Logging Studies $(\mathrm{CB}=6.82)$.

Most central Activities are Measure $(\mathrm{CB}=28.55)$, Record $(\mathrm{CB}=25.46)$, Compare $(\mathrm{CB}=22.55)$ and Analyze $(\mathrm{CB}=18.96)$.

\title{
Conclusions
}

Most frequent edges:

- M/surveystudies - isUsing- I/narrativeitems

- A/report - isPerformedIn-M/laboratorystudies

- A/measure - isSupportedBy- I/narrativeitems

- A/analyze -isPerformedIn- M/laboratorystudies

- A/report - isPerformedIn-M/surveystudies
A strong quantitative perspective in DL evaluation.

Prominence of procedural level classes and properties in the scholarly communication of the DL field. 